\title{
Modifications tissulaires et réactions de défense chez quelques Téléostéens parasités par les Cymothoidae (Crustacés, Isopodes, Hématophages)
}

\author{
par B. ROMESTAND *, M. JANICOT ** et J.-P. TRILLES * \\ * Laboratoire de Physiologie des Invertébrés, Université des Sciences \\ et Techniques du Languedoc, F 34060 Montpellier, Cedex. \\ ** Laboratoire d'Anatomie et Physiologie humaines, \\ Faculté de Pharmacie, F 34000 Montpellier.
}

\section{Résumée.}

Chez les poissons hébergeant des Cymothoidae (Crustacés, Isopodes, Hématophages), des modifications tissulaires sont mises en évidence au niveau du microbiotope parasitaire.

Elles se manifestent principalement par une hypertrophie de l'épiderme et par une profonde désorganisation du tissu conjonctif; en outre, on observe une production importante de mucus à la surface des téguments et un afflux de cellules sanguines (lymphocytes, cellules à granulations éosinophiles) et d'origine histiocytaire (cellules géantes plurinuclées).

\section{Summary.}

Tissular alterations and defence reactions in Cymothoids parasited Teleosteans (Crustacea - Isopoda - blood sucking).

Some tissual alterations of the parasitic microbiotope are shown in fishes harbouring Cymothoidae (blood-sucking Isopods).

These alterations consist mainly in a hypertrophied epidermis and a deeply disturbed conjunctive tissue; moreover, it can be observed an important production of mucus on the tegument surface and a rush of blood cells (lymphocytes, eosenophilic granulated cells) and of cells of histiocytary origin (giant multinucleated cells). 
Etant donné leur régime alimentaire particulier (hématophagie), les Cymothoidae, pour pouvoir se nourrir convenablement, percent les téguments des poissons. Au niveau des lésions ainsi produites par les pièces buccales des parasites, interviennent alors peut-être des modifications tissulaires et des réactions de défense des hôtes parasités ?

Jusqu'à présent, une seule étude, celle de Pflugfelder (1955) sur Crenilabrus cinereus parasité par Anilocra mediterranea (syn. Anilocra frontalis Edwards, 1840), a essayé de répondre à cette question. Il était donc intéressant de poursuivre des recherches dans ce sens et nous exposons aujourd'hui les premiers résultats obtenus dans ce domaine.

\title{
Matériel et méthodes
}

Notre étude a été réalisée sur :

- Des poissons parasités par les Cymothoadiens « de surface»:

- Anilocra physodes (L., 1758) qui parasite préférentiellement Boops boops (L., 1758) (Sparidae, Bogue) ; Maena maena (L., 1758) (Maenidae, Vernière) et Pagellus erythrinus L., 1758 (Sparidae, Pageot).

- Nerocila orbignyi qui se fixe surtout sur les nageoires ou l'appendice caudal des poissons du genre Mugil (Mugilidae, Muge).

- Des poissons parasités par le Cymothoadien buccal Meinertia oestroides (Risso, 1826) qui vit sur le plancher buccal et plus précisément sur la langue des Bogues.

- Des poissons parasités par le Cymothoadien branchial Mothocya epimerica Costa, 1851 qui parasite les Athérines (Atherinidae).

Les poissons ont été récoltés à Sète ou à Agde (Hérault, France) à l'arrivée des chaluts ou à la Senne-de-plage.

Toutes les pièces ont été fixées dans le liquide de Bouin: pour les appendices caudaux des Muges, une décalcification préalable à l'acide formique est nécessaire (R. Martoja et M. Martoja, 1967).

Des coupes à $6 \mu$ ont été réalisées puis colorées à l'hémalum éosine ou à l'hémalum picro-indigo-carmin.

\section{Résultats}

\author{
A. - Modifications tégumentaires chez les poissons parasités par les Cymothoadiens \\ «DE SURFACE 》. \\ $1^{\circ}$ Le tégument des poissons non parasités (Pl. I; photos 1 et 2):
}

La peau des Téléostéens comprend classiquement un épiderme et un derme, ce 

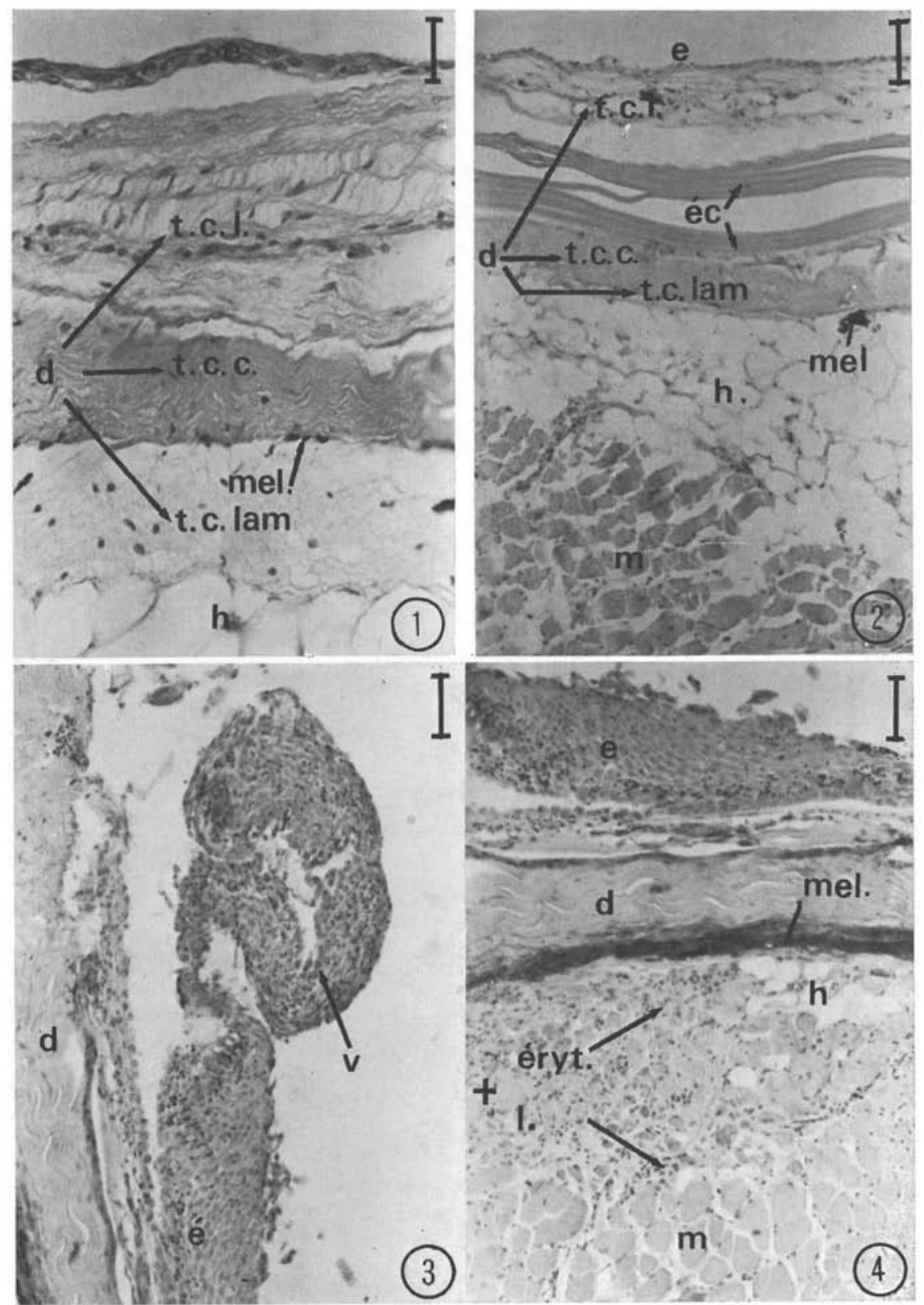

Planche I. - Photo 1: Téguments de Boops boops non parasitée, G $\times 250$. Photo 2 : Téguments de Maena maena non parasitée, $\mathrm{G} \times 100$. Photo 3 : Epiderme hypertrophié (verrue) de Boops boops parasitée par Anilocra physodes, $\mathrm{G} \times 100$. Photo 4: Téguments de Boops boops parasitée par Anilocra physodes; zone proche de la fixation parasitaire, $\mathrm{G} \times 100$. d.: derme ; e.: épiderme éc.: écaille éryt. : érythrocytes; h.: hypoderme ; 1 : lymphocytes ; m. : muscles; mel. : mélanophores; t.c.c. : tissu conjonctif compact ; t.c.l. : tissu conjonctif lâche; t.c.lam. : tissu conjonctif lamelleux; v.: verrue.

Echelles : $1 \mathrm{~cm}=50 \mu$ pour $\mathrm{G} \times 100 ; 1 \mathrm{~cm}=20 \mu$ pour $\mathrm{G} \times 250 ; 1 \mathrm{~cm}=12,5 \mu$ pour $\mathrm{G} \times 400$. 

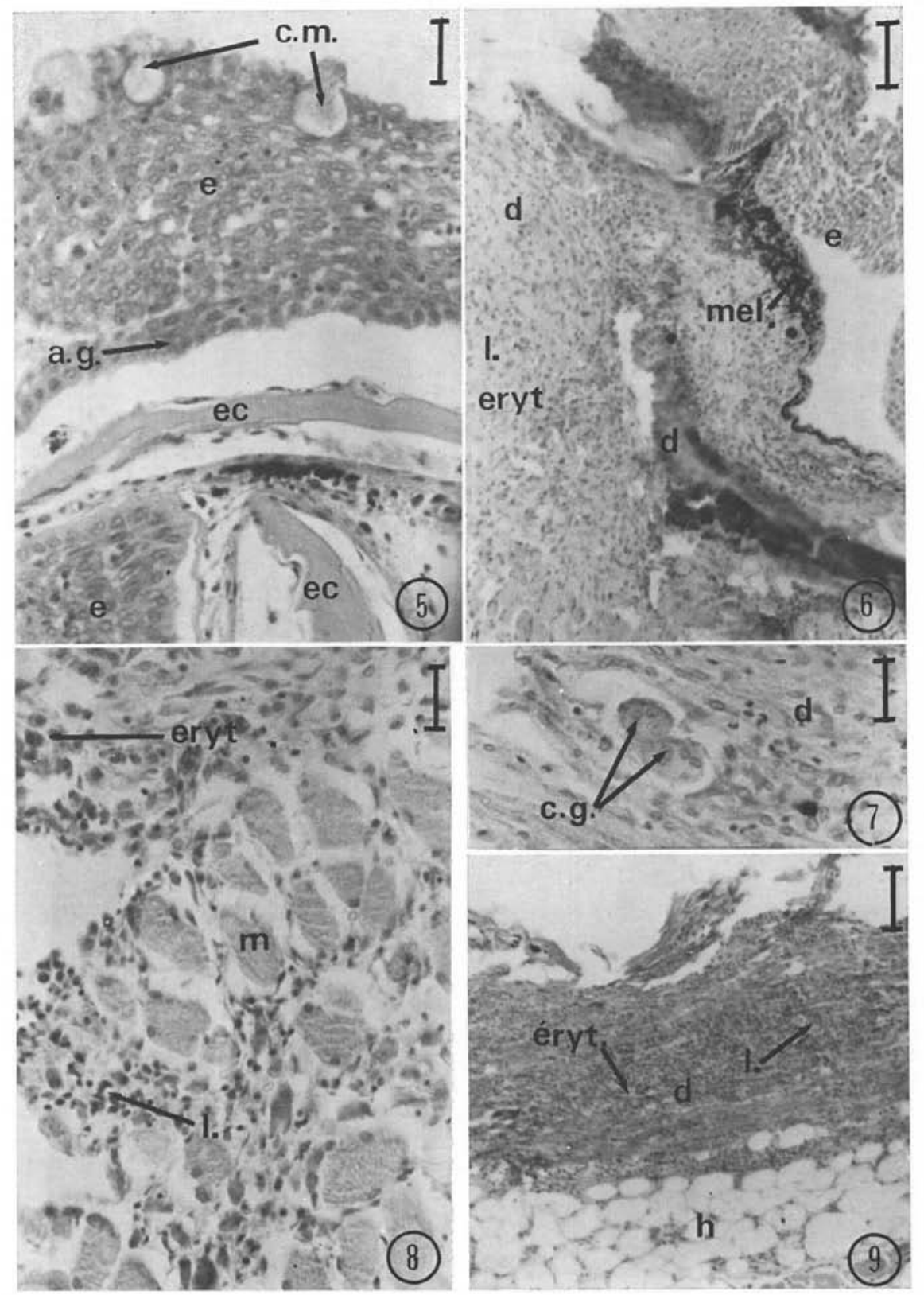

Planche II. - Photo 5: Epiderme hypertrophié de Pagellus erythrinus parasité par Anilocra physodes, $\mathrm{G} \times 250$. Photo 6: Téguments de Boops boops parasitée par Anilocra physodes; zone de fixation du parasite, $\mathrm{G} \times 100$. Photo 7 : Cellules géantes dans le derme de Pagellus erythrinus parasité par Anilocra physodes, $\mathrm{G} \times 250$. Photo 8: Muscles de Maena maena parasitée par Anilocra physodes, $\mathrm{G} \times 250$. Photo 9: Téguments de Maena maena parasitée par Anilocra physodes G $\times 100$.

a.g. : assise génératrice; c.g.: cellule géante; c.m.: cellule muqueuse; d.: derme; é. : épiderme ; éc. : écaille ; éryt. : érythrocytes ; h. : hypoderme ; 1 . : lymphocytes ; m. : muscles.

Echelles: $1 \mathrm{~cm}=50 \mu$ pour $\mathrm{G} \times 100 ; 1 \mathrm{~cm}=20 \mu$ pour $\mathrm{G} \times 250 ; 1 \mathrm{~cm}=12,5 \mu$ pour $\mathrm{G} \times 400$. 
dernier surmontant l'hypoderme et les muscles (Bertin, 1958), (Junqueira et coll., 1970).

L'épiderme est de type Malpighien à évolution cornée; l'assise génératrice repose sur une membrane basale plus ou moins visible suivant les espèces.

Le derme (partie la plus épaisse de la peau) peut être subdivisé en trois couches:

- Une couche de tissu conjonctif lâche avec une grande variété cellulaire, une extrême abondance de vaisseaux sanguins et quelques mélanophores. Il s'agit de la couche nourricière de la peau.

- Une couche de tissu conjonctif compact où les fibres conjonctives sont mêlées à des fibres élastiques distribuées régulièrement en lames parallèles.

- Une couche de tissu conjonctif lamelleux assez réduite, avec quelques mélanophores. Elle surmonte directement l'hypoderme et les muscles.

L'épaisseur de ces différentes couches tégumentaires varie selon les espèces étudiées; ainsi, l'épiderme est plus mince chez les Pageots et les Vernières; le tissu conjonctif lamelleux est très réduit, même parfois inexistant, chez les Vernières, les Muges et les Pageots.

\section{$2^{\circ}$ Le tégument des poissons parasités (Pl. I-II; photos 3 à 9) :}

Si l'on excepte le cas de la Vernière qui voit son épiderme disparaître au niveau de l'emplacement de l'Anilocre, chez les poissons parasités par les Cymothoidea « de surface », on peut observer:

- Une hypertrophie nette de l'épiderme avec, en surface, abondance de volumineuses cellules muqueuses.

Les différentes couches de cellules épidermiques sont envahies par des cellules sanguines (1) (érythrocytes, cellules éosinophiles et lymphocytes). La membrane basale et l'assise génératrice s'interrompent uniquement au niveau de la zone de fixation du parasite.

- Une désorganisation des différentes couches du derme (les strates ne sont plus visibles) et, comme pour l'épiderme, un afflux massif de cellules sanguines. Ça et là, on note la présence de cellules plurinuclées, appelées par Pflugfelder (1955) « cellules à corps étrangers * (Riesenzellen).

- Une importante infiltration de cellules sanguines, plus profondément, au niveau de l'hypoderme et des muscles.

Les écailles, dérivés dermiques recouvertes d'épiderme, sont encore visibles chez les Bogues, les Pageots et les Muges; mais elles semblent disparaître complètement chez les Vernières. Ceci est peut-être en relation avec la minceur des téguments chez Maena maena.

(1) Nous avons établi (résultats non encore publiés) une nomenclature des cellules sanguines rencontrées chez ces diverses espèces de poissons. Six variétés cellulaires ont été distinguées: hémoblastes, érythrocytes, cellules à noyau plurilobé, cellules à granulations éosinophiles, lymphocytes et thrombocytes. 


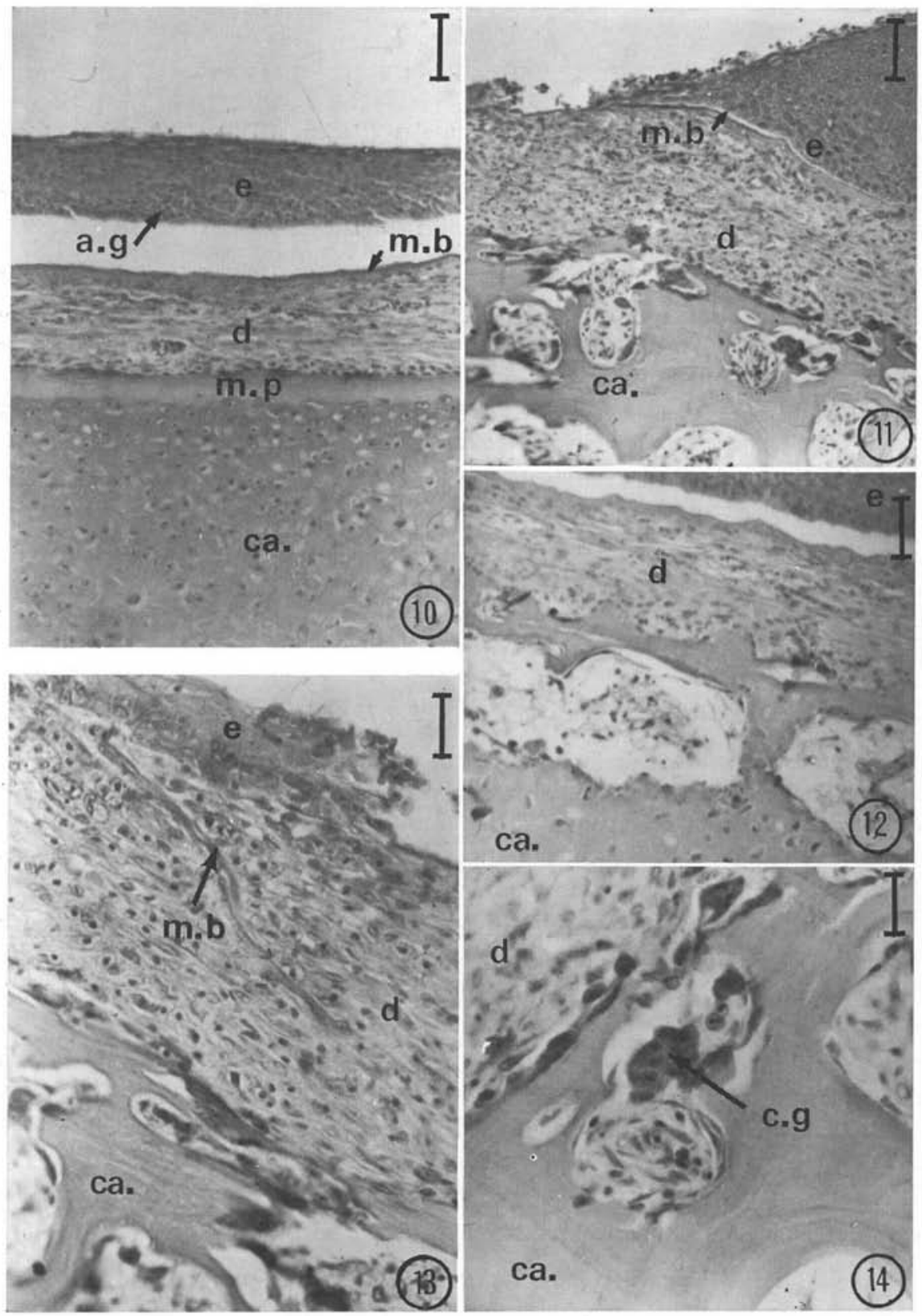

Planche III. - Photo 10: Paroi linguale de Boops boops non parasitée, G $\times 100$. Photo 11: Paroi linguale de Boops boops parasitée par Meinertia astroides; zone de fixation du parasite, $\mathrm{G} \times 100$. Photo 12: Derme et cartilage de Boops boops parasitée par Meinertia astroides; zone assez éloignée de la fixation du parasite; début de dégradation du cartilage, $\mathrm{G} \times 100$. Photo 13: Paroi linguale de Boops boops parasitée par Meinertia cestroides; zone de fixation du parasite, $\mathrm{G} \times 250$. Photo 14: Cartilage lingual de Boops boops parasitée par Meinertia astroides, $\mathrm{G} \times 400$.

a.g. : assise génératrice ; ca. : cartilage ; c.g. : cellule géante ; d. : derme ; e. : épiderme ; m.b.: membrane basale; m.p. : membrane périchondrale.

Echelles : $1 \mathrm{~cm}=50 \mu$ pour $\mathrm{G} \times 100 ; 1 \mathrm{~cm}=20 \mu$ pour $\mathrm{G} \times 250 ; 1 \mathrm{~cm}=12,5 \mu$ pour $\mathrm{G} \times 400$. 

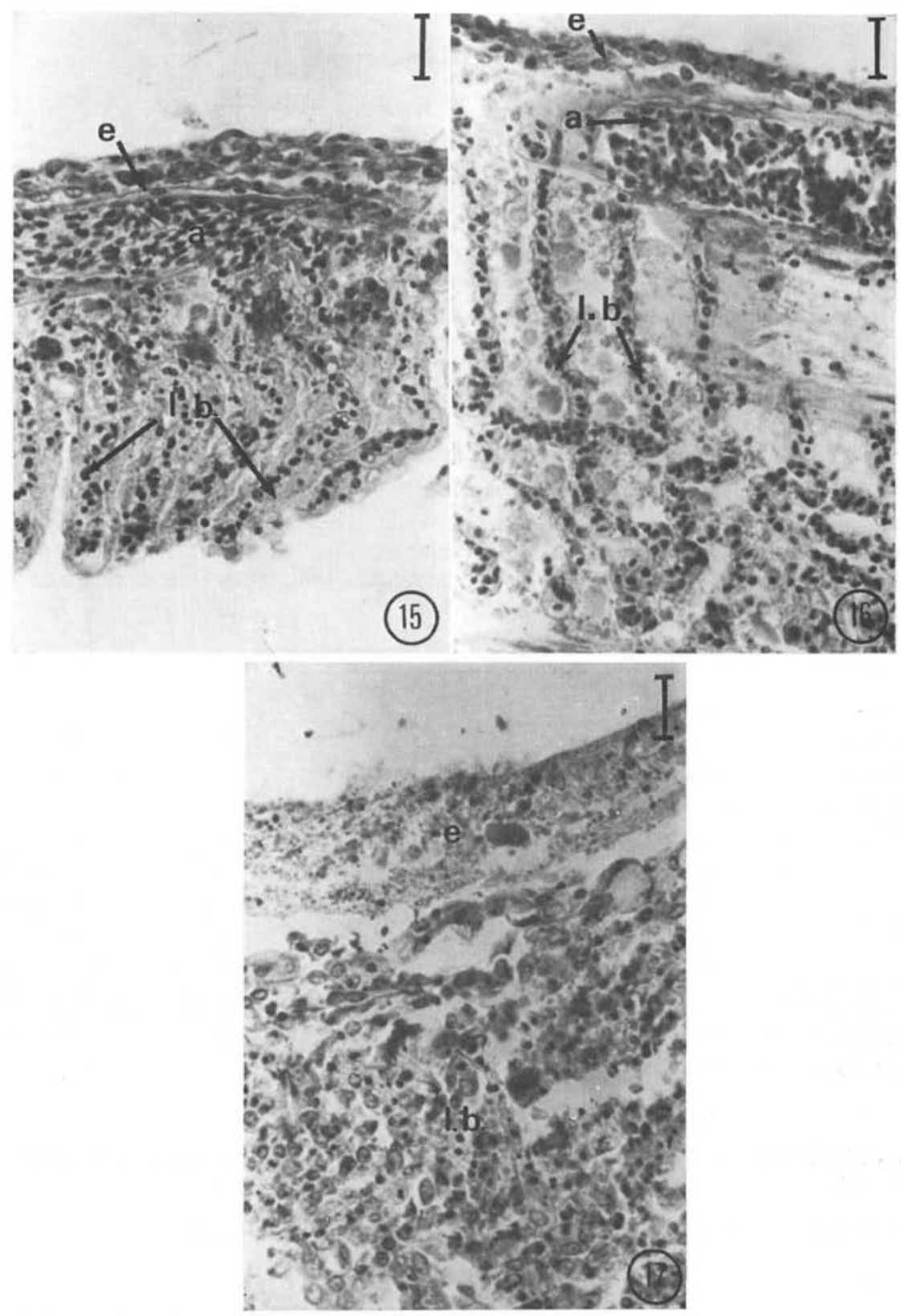

Planche IV. - Photo 15: Lamelles branchiales d'Atherina mochon non parasitée, $\mathrm{G} \times 100$. Photo 16: Lamelles branchiales d'Atherina mochon non parasitée, $\mathrm{G} \times 250$. Photo 17 :

Lamelles branchiales d'Atherina mochon parasitée par Mothocya epimerica, G $\times 250$. a. : artériole; e. : épiderme; 1.b.: lamelles branchiales.

Echelles : $1 \mathrm{~cm}=50 \mu$ pour $\mathrm{G} \times 100 ; 1 \mathrm{~cm}=20 \mu$ pour $\mathrm{G} \times 250 ; 1 \mathrm{~cm}=12,5 \mu$ pour $\mathrm{G} \times 400$. 
B. - Modifications de la paroi linguale des Bogues parasitées par Meinertia astroides.

$1^{\circ}$ La paroi linguale des Bogues non parasités (PI. III ; photo 10 ) :

Chez les Bogues non parasitées̀, la paroi linguale comprend:

- Un épiderme pluristratifié, constitué par 8 à 10 couches cellulaires; la membrane basale et l'assise génératrice sont bien visibles. A la partie supérieure de l'épiderme, on trouve toujours quelques lamelles cornées provenant de la kératinisation des cellules.

- Un derme comportant trois couches bien distinctes:

- Une couche de tissu conjonctif lâche présentant de nombreuses cellules et très irriguée grâce à de nombreux capillaires sanguins.

- Une couche de tissu conjonctif compact, avec une nette prédominance de fibres.

- Une couche de tissu conjonctif lamelleux, le périchondre, avec une nette prépondérance cellulaire et très vascularisée. Elle doit jouer un rôle dans l'accroissement par apposition du cartilage lingual (cartilage hyalin) où chondroplastes et chondroblastes sont bien visibles dans la substance fondamentale.

$2^{\circ}$ La paroi linguale des Bogues parasitées (Pl. III; Photo 11 à 14):

- Au niveau du point d'ancrage du parasite, l'épiderme subit une réduction très importante, puis disparaît totalement (l'assise génératrice, ainsi que la membrane basale); il est par contre légèrement hypertrophié de part et d'autre de la zone de fixation parasitaire.

- Au niveau du derme, il n'est plus possible de distinguer les différentes couches. Il se produit une désorganisation de tout le tissu conjonctif (il n'est pas rare d'y rencontrer des restes de membrane basale) et il est alors envahi par des cellules sanguines (érythrocytes, cellules éosinophiles, lymphocytes et cellules plurinucléées). Le cartilage paraît envahi par le tissu conjonctif et on ne retrouve plus sa structure classique; les chondroblastes et les chondroplastes disparaissent; la substance fondamentale se creuse de cavités dans lesquelles on peut mettre en évidence de très nombreuses cellules géantes.

C. - Modifications des branchies chez les Athérines parasitées par Mothocya epimerica.

$1^{\circ}$ Chez les poissons non parasités (Pl. IV; Photo 15-16):

Les filaments branchiaux, revêtus d'un épithélium simple pavimenteux, sont constitués par une lame conjonctive dans laquelle pénètrent les artérioles afférentes et efférentes, ainsi que des filets nerveux.

Les lamelles branchiales que portent les filaments branchiaux ont une structure beaucoup plus simple que ces derniers. Elles sont réduites à deux couches épithéliales et renferment de nombreuses cellules sanguines. 
$2^{\circ}$ Chez les poissons parasités (Pl. IV; Photo 17):

On observe un blanchiment et une atrophie des filaments branchiaux en arrière du point de fixation du parasite.

Les coupes histologiques montrent un épiderme légèrement hypertrophié, tandis que les lamelles branchiales, vidées de leur contenu sanguin, sont très aplaties.

Par la suite, les tissus n’étant plus irrigués, se nécrosent.

En amont du point de fixation du parasite, on peut observer des amas de globules rouges dus à la stase sanguine.

\section{Conclusions}

Le parasitisme par les Cymothoadiens est donc suivi de modifications tissulaires plus ou moins intenses au niveau des lieux de fixation des parasites; elles paraissent correspondre à des réactions de défenses locales des poissons hôtes.

Quelle que soit l'espèce considérée (mais à un degré moindre pour Mothocya epimerica), ces modifications se manifestent par :

- Une hypertrophie de l'épiderme, toujours accompagnée, au point d'impact du parasite, d'une «dissolution » de la membrane basale et d'une disparition de l'assise génératrice.

- Une profonde désorganisation du tissu conjonctif sous-jacent avec en plus, dans le cas particulier de la Bogue, une destruction du cartilage lingual. Il se produit d'ailleurs très certainement un changement dans la structure chimique de la substance cartilagineuse fondamentale.

Ces modifications sont en outre associées à :

- Une production importante de mucus à la surface de l'épiderme, en particulier dans le cas des Bogues, des Pageots et des Muges parasités par les Cymothoadiens de surface.

- Un afflux de cellules sanguines, surtout de cellules à granulations éosinophiles et de lymphocytes. On observe également la présence de cellules géantes plurinuclées d'origine histiocytaire.

Ces résultats sont en accord avec ceux de Pflugfelder (1955). L'hôte paraît réagir contre le parasite en particulier par des modifications tégumentaires, une production de mucus et vraisemblablement une réaction immunologique mettant en jeu des cellules lymphocytaires et des cellules d'origine histiocytaire. Ce dernier type de réaction pourrait être en relation avec les résultats déjà obtenus chez ces animaux (Romestand et Trilles, 1975). 


\section{Bibliographie}

Bertin (L.), 1958. - Peau et pigmentation, in: Traité de Zoologie de Grasse, Anatomie Systématique - Biologie. Agnathes et poissons. Zoologie, T. 13, pp, 433-458 (A). Masson, édit., Paris.

Grasse (P.-P.) et Devillers (C.), 1965. - Précis de Sciences biologiques, Zoologie II. Vertébrés. Masson, édit., Paris, 1129 p.

Junqueira (L. C. U.), Tolédo (A. M. S.) et Porter (K. P.), 1970. - Observations on the skin of the Teleost Fundulus heteroclitus (L.). Arch. histol. Jap., 32, 1-15.

Martoja (R.) et Martoja (M.), 1967. - Initiation aux techniques de l'histologie animale. Masson, édit., Paris, 345 p.

Pflugfelder (O.), 1955. - Abwehrreaktionen von Crenilabrus cinereus V.C.R.S. bei befall durch Anilocra mediterranea Leach, Z. Parasitenkunde, 17, S., 122-130.

Romestand (B.) et Trilles (J.-P.), 1975. - Les relations immunologiques, "hôte-parasite » chez les Cymothoidea (Isopoda, Flabellifera). C.R. Acad. Sci., 280, Sér. D, 21712173.

TriLles (J.-P.), 1968. - Recherches sur les Isopodes Cymothoidea des côtes françaises. Thèse d'Etat, Université Montpellier, $\mathrm{N}^{\circ}$ A.O. 2305. 